\title{
Prevalence and Prognostic Significance of Extramural Venous Invasion in Patients with Locally Advanced Esophageal Cancer
}

\author{
Zohra Faiz, MD ${ }^{1}$, Lotte J. W. Huijgen, MD ${ }^{2}$, H. J. Alqethami, BSc ${ }^{2}$, J. G. M. Burgerhof, MSc ${ }^{3}$, \\ Gursah Kats-Ugurlu, MD, $\mathbf{P h D}^{2}$, and John T. M. Plukker, MD, PhD ${ }^{1}$ \\ ${ }^{1}$ Department of Surgery, University of Groningen, University Medical Center Groningen, Groningen, The Netherlands; \\ ${ }^{2}$ Department of Pathology, University of Groningen, University Medical Center Groningen, Groningen, The Netherlands; \\ ${ }^{3}$ Department of Epidemiology, University of Groningen, University Medical Center Groningen, Groningen, The \\ Netherlands
}

\begin{abstract}
Background. Extramural venous invasion (EMVI) is a known adverse prognostic factor in patients with colorectal carcinoma. The prevalence and significance of EMVI in esophageal cancer (EC) patients is still unclear.

Methods. From a prospectively maintained database, we retrospectively reviewed the resection specimens of patients with pathologic locally advanced (pT3/T4/N0-3) EC who were treated with curative intent between 2000 and 2015. Patients with previous malignancies and gastroesophageal junction (type II/III) tumors were excluded. Included were 81 patients who underwent surgery alone and 37 patients who underwent neoadjuvant chemoradiotherapy (nCRT). EMVI was assessed on hematoxylin and eosin slides and confirmed or excluded by additional Elastica van Gieson staining. Survival was analyzed using a multivariable Cox regression.

Results. EMVI was present in $23.5 \%(n=19)$ of patients in the surgery-alone group and $21.6 \%(n=8)$ of patients in the nCRT group. The prevalence of EMVI after surgery alone was significantly high in squamous cell carcinomas and among tumors located in the mid-esophagus, as well as those with lymphovascular invasion $(p<0.05)$. After nCRT, the presence of EMVI was significantly high in
\end{abstract}

Part of this study was presented at ASCO-GI 2017 and ECCO/ESSO 2017.

(C) The Author(s) 2018

First Received: 8 May 2017;

Published Online: 2 April 2018

J. T. M. Plukker, MD, PhD

e-mail: j.t.m.plukker@umcg.nl tumors with lymphovascular and perineural tumor growth $(p=0.034)$. EMVI status was an independent adverse prognostic factor for disease-free survival [hazard ratio (HR) 7.0, 95\% confidence interval (CI) 2.3-21.8; $p=0.001$ ] and overall survival (HR 6.5, 95\% CI 2.2-19.1; $p=0.001)$ in the surgery-alone group for node-positive tumors.

Conclusions. In this study of locally advanced $>$ pT3/N03 EC patients, EMVI was present in $23.5 \%$ of patients in the surgery-alone group and in $21.6 \%$ of patients after nCRT. EMVI was an independent adverse prognostic factor in patients after surgery alone.

Invasion of tumor cells into blood vessels is an important expression of the metastatic potency of malignant tumors. In esophageal cancer (EC), distant metastases are common and seem to be associated with venous invasion (VI). ${ }^{1,2}$ Current TNM classifications recognize lymphovascular invasion (LVI) as a prognostic factor. ${ }^{3,4}$ Hence, it may be important to report the type of vascular invasion (VI) of tumor cells during routine pathologic workup in EC. Both the Association of Directors of Anatomic and Surgical Pathology (ADASP) and the College of American Pathologists (CAP) stress that extramural venous invasion (EMVI) is an independent predictor of poor prognosis in colorectal cancer (CRC). ${ }^{5,6}$ EMVI is defined as the microscopic presence of tumor cells in venous blood vessels beyond the muscularis propria (Fig. 1).$^{6-11}$

The prevalence of EMVI in CRC resection specimens is approximately $28 \%{ }^{6}$ In contrast to CRC, the prevalence and prognostic significance of EMVI in EC has not been well studied. ${ }^{9}$ In general, most of the studies in EC were performed to differentiate VI from lymphatic invasion. 


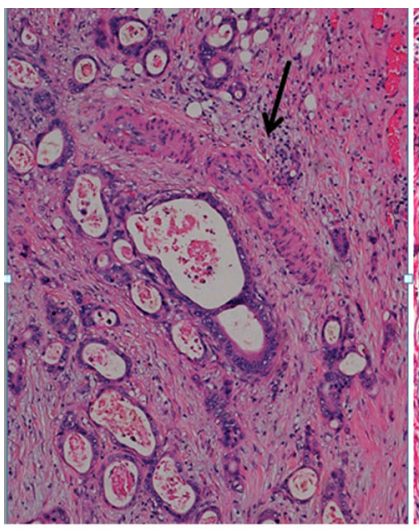

A

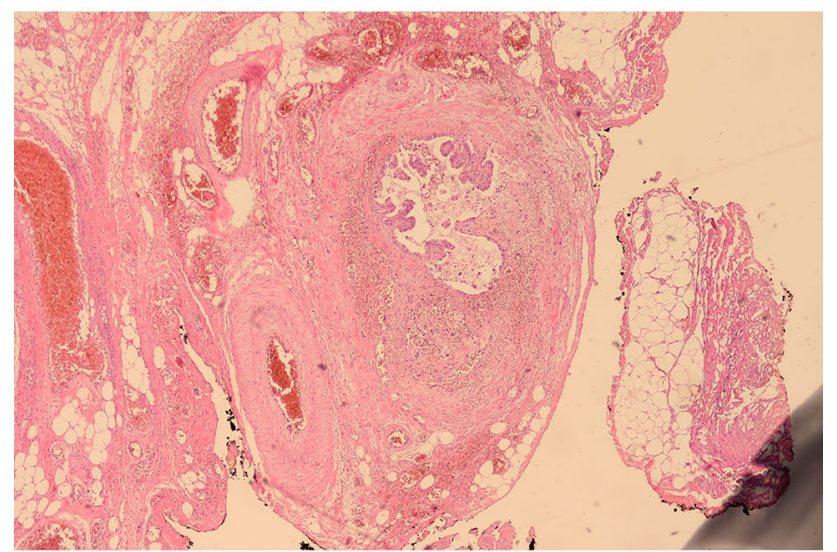

A1

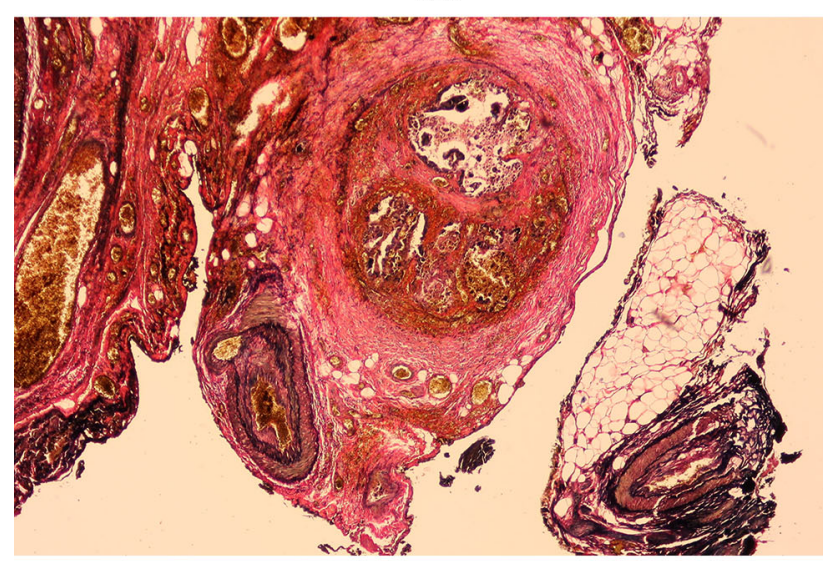

B1

FIG. 1 'Orphan artery' sign (arrow) suspicious for extramural venous invasion identified on a hematoxylin and eosin slide in esophageal cancer resection (A/A1). The deeper section of the corresponding tumor specimen stained with Elastica van Gieson shows the black-stained elastic fibers around the vein (arrowheads) next to the artery (arrow), confirming the presence of tumor cells in an extramural vein $(\mathrm{B} / \mathrm{B} 1)$

Presently, neither the Royal College of Pathologists (RCP) nor the CAP protocol requires differentiation of intramural VI (IMVI), EMVI, and lymphatic invasion in esophageal resection specimens. ${ }^{9}$ To this end, the objectives of our study were to determine the prevalence and assess the prognostic significance of EMVI, confirmed or excluded by Elastica van Gieson (EVG) staining of the resection specimens in patients with pathological T3 or higher EC treated by surgery alone, and those with neoadjuvant chemoradiotherapy (nCRT) followed by surgery.

\section{MATERIALS AND METHODS}

\section{Patients}

EMVI was retrospectively analyzed on prospectively collected data of 182 consecutive patients with a curative resectable EC who underwent surgery alone between 2000 and 2014. Excluded were 98 patients with a pathological stage $\leq \mathrm{T} 1-\mathrm{T} 2$ EC and/or cardia-gastroesophageal junction (GEJ; type II/III) tumors, which are frequently considered as gastric cancer. All patients were aged $\geq 18$ years and were staged according to a standard protocol with 18F-fluorodeoxyglucose positron emission tomography (FDG-PET), computed tomography (CT), and endoscopic ultrasonography (EUS). To evaluate the impact of EMVI after current treatment with nCRT plus surgery according to the CROSS (ChemoRadiotherapy for Oesophageal cancer followed by Surgery Study), ${ }^{12}$ we also examined 37 of the 54 patients with a pathological T3 EC who met the same inclusion criteria, with the exception of treatment with nCRT between 2012 and 2015. This study was performed according to the rules of the National Health Care, with approval from the Institutional Ethics Board.

\section{Methods}

Histopathology Assessment For each patient, archival slides of the tumor resection specimens were reviewed by two gastrointestinal pathologists. EMVI was scored as the presence of tumor cells within venous structures beyond the muscularis propria, as characterized by the 'protruding tongue' and the 'orphan artery' signs. The 'protruding tongue' sign is defined as a tongue-like protrusion of tumor extending from the deepest invasive front into the surrounding peri-esophageal fat, ${ }^{9}$ and the 'orphan artery' sign is observed when tumor invasion is present as a circumscribed tumor nodule near a muscularized artery without invading accompanying veins on hematoxylin and eosin (H\&E) slides (Fig. 1). ${ }^{6,13}$ In all EMVI suspected cases on H\&E slides, additional EVG staining was performed to confirm or exclude the presence of EMVI. The elastic lamina within medium to large vessels can be seen as black elastic fibers in EVG-stained slides, which 
can distinguish lymphatic vessels from veins. EVG staining was performed according to a standard protocol. ${ }^{11}$ Slides of 4-5 $\mu \mathrm{m}$ were performed from the corresponding tumor in paraffin blocks. One to three tumor slides from cases suspicious for EMVI were additionally stained with EVG.

Other examined pathological parameters were $\mathrm{T}$ stage (T3/T4), N stage, tumor length, histologic type, tumor differentiation grade (well/moderate vs. poor/signet cell/mucinous), perineural invasion (PNI), and circumferential resection margin (CRM) involvement. PNI was defined as tumor cell infiltration in any layer of the nerve sheath, while both LVI and VI were defined as the presence of tumor cells within an endothelium-lined space without underlying muscular walls. CRM was assessed according to the RCP definition, which defines microscopic tumor cells within $1 \mathrm{~mm}$ of the $\mathrm{CRM}$ as involved (R1 resection).

Follow-Up Patients were evaluated postoperatively every 3 months for the first year, every 6 months for the second and third years, and yearly thereafter. If recurrence was clinically suspected, patients underwent CT or PET/ CT scans of the chest/abdomen, and endoscopy for confirmation of locoregional recurrence. Recurrent disease was either proven cytologically/histologically or when unequivocally present on radiologic imaging.

Statistics Fisher's exact test was used to compare characteristics of patients with and without EMVI, and the log-rank test was used for univariable survival analyses of disease-free (DFS) and overall survival (OS). DFS was defined as the time from surgery to the date of recurrence or death. The prognostic value of different factors was first examined in univariable analyses. Clinically relevant factors with a $p$ value $<0.2$ in univariable analysis were included in the Cox regression multivariable analysis. Survival curves were calculated using the Kaplan-Meier method. Patients were censored at the last point of known contact during follow-up without acquiring the outcomes of interest. The backward conditional Cox regression model was used to delineate significant prognostic factors for survival. Hazard ratios (HR) and 95\% confidence intervals (CI) were generated, and a $p$ value $<0.05$ was considered significant in the multivariable analysis. Statistical analysis was performed using SPSS 20.0 software (IBM Corporation, Armonk, NY, USA).

\section{RESULTS}

\section{Patient and Tumor Characteristics}

Surgery-Alone Group Of the 84 included patients with pathological $\geq \mathrm{T} 3 \mathrm{EC}$, EMVI was suspected in 47 patients
(55.9\%) on review of H\&E-stained slides. In three suspicious EMVI cases, EVG staining could not be performed due to the loss of the area of interest in additional slides, and these patients were therefore excluded from further analysis. The median age of the remaining 81 patients was 68 years (range 50-85). Tumornegative CRM of $>1 \mathrm{~mm}$ (R0) was seen in 42 (51.9\%) patients, and EMVI could be confirmed on EVG-stained slides in $19(23.5 \%)$ cases. The relation of EMVI with the examined tumor characteristics are presented in Table 1. The presence of EMVI was significantly high in tumors located in the mid-esophagus $(57.1 \%$ vs. $16.4 \%$ in the distal esophagus; $p=0.003$ ) and in squamous cell carcinomas [SSC; $42.9 \%$ vs. $16.7 \%$ in adenocarcinoma (AC); $p=0.033]$. Moreover, the presence of EMVI was significantly higher in patients with LVI-positive tumor $(p=0.006)$. EMVI showed no relationship with pathological (p)T stage and $\mathrm{pN}$ stage, tumor length or differentiation grade, or with PNI or CRM involvement (Table 1).

Neoadjuvant Treatment Group Of the 37 patients with pathological T3 EC enrolled in this study, EMVI was suspected in $19(51.4 \%)$ patients on review of H\&E-stained slides (Table 2). Tumor-negative CRM of $>1 \mathrm{~mm}$ (R0) was seen in 33 patients (89.2\%), and EMVI could be confirmed on EVG-stained slides in 8 of the 19 cases $(42.1 \%)$. In relation to the examined tumor characteristics (Tables 3, 4), EMVI was only significant in tumors with LVI and perineural tumor growth $(p=0.034)$.

\section{Survival Analysis}

Surgery-Alone Group Follow-up data were available in 74 of the 81 examined patients. Excluded from survival analysis were six patients who died $<90$ days after surgery and one patient with a simultaneous colon carcinoma. In the remaining 74 resected esophageal tumors, EMVI was confirmed in 17 of 37 (46\%) suspected cases. Median DFS ( 22 vs. 22 months; $p=0.297$ ) and OS (23 vs. 26 months; $p=0.226$ ) were not different among patients with or without EMVI. Distant recurrence-free survival was also not different in both groups ( 22 vs. 25 months; $p=0.306$ ).

In the univariable analysis (Table 2), independent prognostic factors associated with DFS were gender, pT3 stage, pN1-3, positive CRM and EMVI/N (nodal status). The backward conditional Cox regression multivariable analysis (Table 3) showed that positive CRM (HR 2.4, 95\% CI 1.3-4.6) and EMVI/N (nodal status) [HR 1.8, 95\% CI 1.3-2.7] were independent prognostic factors for DFS. The median DFS for EMVI and nodal status were significantly different: EMVI $-/ \mathrm{N}-$ : 79 months; EMVI +/ 
TABLE 1 Cohort demographics by the presence or absence of EMVI in the surgery-alone group

\begin{tabular}{|c|c|c|c|c|}
\hline Pathological characteristics & Total $[N=81](100 \%)$ & EMVI-positive $[N=19](23.5 \%)$ & EMVI-negative $[N=62](76.5 \%)$ & $p$ value \\
\hline \multicolumn{5}{|l|}{ Localization } \\
\hline Middle & $14(17.3)$ & $8(42.1)$ & $6(9.7)$ & \multirow[t]{2}{*}{0.003} \\
\hline Distal & $67(82.7)$ & $11(57.9)$ & $56(90.3)$ & \\
\hline \multicolumn{5}{|l|}{ Histologic type } \\
\hline $\mathrm{AC}$ & $60(74.0)$ & $10(52.6)$ & $50(80.6)$ & \multirow[t]{2}{*}{$\mathbf{0 . 0 3 3}$} \\
\hline SCC & $21(26.0)$ & $9(47.4)$ & $12(19.4)$ & \\
\hline \multicolumn{5}{|l|}{$\mathrm{T}$ stage } \\
\hline T3 & $74(91.3)$ & $19(100)$ & $55(88.7)$ & \multirow[t]{2}{*}{0.190} \\
\hline $\mathrm{T} 4$ & $7(8.6)$ & $0(0)$ & $7(11.3)$ & \\
\hline \multicolumn{5}{|l|}{$\mathrm{N}$ stage } \\
\hline Present & $59(72.8)$ & $13(68.4)$ & $46(74.2)$ & \multirow[t]{2}{*}{0.769} \\
\hline Absent & $22(27.2)$ & $6(31.6)$ & $16(25.8)$ & \\
\hline \multicolumn{5}{|l|}{ Differentiation grade } \\
\hline Well/moderated & $51(63.0)$ & $15(78.9)$ & $36(58.1)$ & \multirow[t]{2}{*}{0.113} \\
\hline Poor signet/mucinous & $30(37.0)$ & $4(21.0)$ & $26(41.9)$ & \\
\hline \multicolumn{5}{|l|}{ Tumor length, $\mathrm{cm}^{\mathrm{a}}$} \\
\hline$>3$ & $64(81)$ & $18(94.7)$ & $46(76.7)$ & \multirow[t]{2}{*}{0.154} \\
\hline$\leq 3$ & $15(19)$ & $1(5.3)$ & $14(23.3)$ & \\
\hline \multicolumn{5}{|l|}{ Perineural invasion } \\
\hline Present & $51(63.0)$ & $14(73.7)$ & $37(59.7)$ & \multirow[t]{2}{*}{0.537} \\
\hline Absent & $30(37.0)$ & $5(26.3)$ & $25(40.3)$ & \\
\hline \multicolumn{5}{|l|}{ Lymphovascular invasion } \\
\hline Present & $29(35.8)$ & $12(63.2)$ & $17(27.4)$ & \multirow[t]{2}{*}{0.006} \\
\hline Absent & $52(64.2)$ & $7(36.8)$ & $45(72.6)$ & \\
\hline \multicolumn{5}{|l|}{ CRM } \\
\hline Positive, $\leq 1 \mathrm{~mm}$ & $39(48.1)$ & $7(36.8)$ & $32(51.6)$ & \multirow[t]{2}{*}{0.302} \\
\hline Negative, $>1 \mathrm{~mm}$ & $42(51.9)$ & $12(63.2)$ & $30(48.4)$ & \\
\hline
\end{tabular}

Bold values are statistically significant $(p<0.05)$

$C R M$ circumferential resection margin, $E M V I$ extramural venous invasion, $A C$ adenocarcinoma, $S C C$ squamous cell carcinoma

${ }^{\text {a }}$ Two missing values

$\mathrm{N}-: 32$ months; EMVI $-/ \mathrm{N}+: 18$ months; EMVI + /

$\mathrm{N}+: 14$ months (Fig. 2a).

In the univariable analysis (Table 2), independent prognostic factors associated with OS were $\mathrm{pT}, \mathrm{pN}$ stage, CRM and EMVI/N (nodal status). The backward conditional multivariable Cox regression analysis showed that independent prognostic factors for OS were pT stage (HR 3.7, 95\% CI 1.5-9.2) and EMVI/N (nodal status) [HR 1.8, 95\% CI 1.3-2.6] (Table 3). The median OS for EMVI and nodal status were significantly different: EMVI $-/ \mathrm{N}-$ : 81 months; EMVI +/N - : 40 (14-65) months; EMVI -/ $\mathrm{N}+$ : 21 (16-25) months; EMVI +/N + : 14 (10-17) months (Fig. 2b).

When adjusted for histologic type, EMVI was significantly prognostic for DFS in SCCs (HR 5.0, 95\% CI 1.0-23.8; $p=0.043$ ) and for OS in ACs after surgery alone (HR 2.5, 95\% CI 1.1-5.6; $p=0.031$ ) [data not shown].
Neoadjuvant Treatment Group Three of the 37 included patients with pathological T3 EC died $<90$ days after surgery and were excluded from survival analysis. In the remaining 34 esophageal tumors, EMVI was confirmed in 8 of $26(30.7 \%)$ suspected cases. Both DFS (15 vs. 26 months; $p=0.827$ ) and OS (median 17 vs. 34 months; $p=0.954$ ) were not different among patients with or without EMVI. Both the univariable analyses and backward conditional multivariable Cox regression analysis have shown no independent prognostic factors associated with DFS (Tables 5, 6). The median DFS for EMVI and nodal status were not significantly different: EMVI -/N - : 41 months; EMVI +/N - : 13 months; EMVI $-/ \mathrm{N}+: 16$ months; EMVI $+/ \mathrm{N}+: 15$ months after nCRT.

In the univariable analyses, a positive CRM was the only independent prognostic factor associated with OS (HR 3.7, 95\% CI 1.1-12.4) (Table 5). The backward conditional 
TABLE 2 Univariable analyses with regard to DFS and OS in 74 patients with $\geq$ pT3 in the surgery-alone group

\begin{tabular}{|c|c|c|c|c|}
\hline \multirow[t]{2}{*}{ Factor } & \multicolumn{2}{|l|}{ DFS } & \multicolumn{2}{|l|}{ OS } \\
\hline & $\mathrm{HR}(95 \% \mathrm{CI})$ & $p$ value & $\mathrm{HR}(95 \% \mathrm{CI})$ & $p$ value \\
\hline Sex (male/female) & $0.6(0.2-1.2)$ & 0.155 & $0.6(0.3-1.3)$ & 0.227 \\
\hline Age & $0.7(0.3-1.8)$ & 0.548 & $0.9(0.4-2.0)$ & 0.805 \\
\hline Tumor length $(\leq 3 />3 \mathrm{~cm})$ & $0.9(0.9-1.0)$ & 0.291 & $1.0(0.9-1.0)$ & 0.264 \\
\hline Localization (mid/distal) & $0.9(0.6-1.3)$ & 0.721 & $0.9(0.4-1.9)$ & 0.884 \\
\hline Histology (SCC/AC) & $0.7(0.4-1.5)$ & 0.450 & $0.6(0.7-1.3)$ & 0.238 \\
\hline pT stage (T3/T4) & $2.9(1.0-8.3)$ & 0.047 & $4.0(1.6-9.8)$ & 0.002 \\
\hline pN1-3 & $1.7(1.2-2.4)$ & 0.002 & $1.5(1.1-2.1)$ & 0.004 \\
\hline Differentiation grade (good/moderate vs. poor/signet cell/mucinous) & $0.8(0.4-1.5)$ & 0.641 & $0.7(0.4-1.4)$ & 0.367 \\
\hline Perineural growth (present/absent) & $1.3(0.7-2.3)$ & 0.411 & $1.0(0.6-1.8)$ & 0.937 \\
\hline Lymphovascular invasion (present/absent) & $1.1(0.6-2.1)$ & 0.649 & $1.2(0.7-2.1)$ & 0.491 \\
\hline CRM (positive/negative) & $2.4(1.4-4.3)$ & 0.002 & $2.0(1.2-3.4)$ & 0.012 \\
\hline EMVI (present/absent) & $1.4(0.7-2.7)$ & 0.305 & $1.5(0.8-2.7)$ & 0.236 \\
\hline EMVI/N (nodal status) & $1.8(1.3-2.6)$ & 0.001 & $1.8(1.3-2.6)$ & $<\mathbf{0 . 0 0 1}$ \\
\hline EMVI $-/ \mathrm{N}-$ & Ref. & & Ref. & \\
\hline $\mathrm{EMVI}+/ \mathrm{N}-$ & $1.8(0.4-7.7)$ & 0.413 & $1.9(0.4-8.0)$ & 0.377 \\
\hline EMVI $-/ \mathrm{N}+$ & $3.6(1.4-9.3)$ & 0.008 & $3.9(1.5-9.9)$ & 0.005 \\
\hline $\mathrm{EMVI}+/ \mathrm{N}+$ & $6.1(2.0-18.6)$ & 0.002 & $6.3(2.1-18.3)$ & 0.001 \\
\hline
\end{tabular}

Bold indicates $p$ value $<0.2$ in univariable analysis were included in the Cox regression multivariable analysis

$D F S$ disease-free survival, $O S$ overall survival, $p$ pathological, $C R M$ circumferential resection margin, $E M V I$ extramural venous invasion, $H R$ hazard ratio, $C I$ confidence interval, SCC squamous cell carcinoma, $A C$ adenocarcinoma

TABLE 3 Multivariable analyses (backward conditional Cox regression model) with regard to DFS and OS in 74 patients with $\geq$ pT3 esophageal cancer in the surgery-alone group

\begin{tabular}{|c|c|c|c|c|}
\hline \multirow[t]{2}{*}{ Factor } & \multicolumn{2}{|l|}{ DFS } & \multicolumn{2}{|l|}{ OS } \\
\hline & HR $(95 \%$ CI $)$ & $p$ value & HR (95\% CI) & $p$ value \\
\hline Sex & $1.0(10.0-1.0)$ & 0.983 & - & \\
\hline pT stage (T3 vs. T4) & $2.1(0.7-6.2)$ & 0.183 & $3.7(1.5-9.2)$ & 0.006 \\
\hline pN1-3 & $0.9(0.5-1.7)$ & 0.793 & $0.7(0.4-1.3)$ & 0.210 \\
\hline CRM [positive/negative] & $2.4(1.3-4.6)$ & 0.005 & $1.5(0.9-2.7)$ & 0.156 \\
\hline EMVI/N (nodal status) & $1.8(1.3-2.7)$ & 0.001 & $1.8(1.3-2.6)$ & $<\mathbf{0 . 0 0 1}$ \\
\hline EMVI -/N - & Ref. & & Ref. & \\
\hline $\mathrm{EMVI}+/ \mathrm{N}-$ & $2.5(0.6-11.1)$ & 0.214 & $1.9(0.5-8.2)$ & 0.364 \\
\hline EMVI $-/ \mathrm{N}+$ & $3.0(1.1-7.8)$ & 0.024 & $3.5(1.3-9.1)$ & 0.010 \\
\hline $\mathrm{EMVI}+/ \mathrm{N}+$ & $7.0(2.3-21.8)$ & 0.001 & $6.5(2.2-19.1)$ & 0.001 \\
\hline
\end{tabular}

Bold values are statistically significant $(p<0.05)$

$D F S$ disease-free survival, $O S$ overall survival, $p$ pathological, $C R M$ circumferential resection margin, $E M V I$ extramural venous invasion, $H R$ hazard ratio, $C I$ confidence interval

Cox regression multivariable analysis has shown that nodal involvement (HR 1.6, 95\% CI 1.1-2.5) was the only independent prognostic for OS (Table 6). The median OS for EMVI and nodal status were not different: EMVI -/ $\mathrm{N}-: 37$ months; EMVI +/N - : 15 months; EMVI -/ $\mathrm{N}+: 18$ months; EMVI $+/ \mathrm{N}+: 17$ months after nCRT.

\section{DISCUSSION}

In EC, the prevalence and significance of EMVI has not been well studied. In this study, EMVI was present in approximately one-quarter of patients with a pathologically staged T3 or higher EC after surgery alone, and in $21.6 \%$ of patients after nCRT. The rate of EMVI in the surgery-alone group was higher in mid-esophageal carcinomas $(42.1 \%$ vs. 
TABLE 4 Cohort demographics by the presence or absence of EMVI in the neoadjuvant chemoradiotherapy group

\begin{tabular}{|c|c|c|c|c|}
\hline Pathological characteristics & Total $[N=37](100 \%)$ & EMVI-positive $[N=8](21.6 \%)$ & EMVI-negative $[N=29](78.4 \%)$ & $p$ value \\
\hline \multicolumn{5}{|l|}{ Localization } \\
\hline Middle & $1(2.7)$ & $0(0)$ & $1(3.4)$ & \multirow[t]{2}{*}{0.784} \\
\hline Distal & $36(97.3)$ & $8(100)$ & $28(96.6)$ & \\
\hline \multicolumn{5}{|l|}{ Histologic type } \\
\hline $\mathrm{AC}$ & 35 (94.6) & $7(87.5)$ & $28(96.6)$ & \multirow[t]{2}{*}{0.390} \\
\hline SCC & $2(5.4)$ & $1(12.5)$ & $1(3.4)$ & \\
\hline \multicolumn{5}{|l|}{$\mathrm{T}$ stage } \\
\hline $\mathrm{T} 3$ & $37(100)$ & $8(100)$ & $29(100)$ & - \\
\hline \multicolumn{5}{|l|}{$\mathrm{N}$ stage } \\
\hline Present & 17 (45.9) & $4(50)$ & $13(44.8)$ & \multirow[t]{2}{*}{0.553} \\
\hline Absent & $20(54.1)$ & $4(50)$ & $16(55.2)$ & \\
\hline \multicolumn{5}{|l|}{ Differentiation grade } \\
\hline Well/moderated & $23(62.2)$ & $5(62.5)$ & $18(62.1)$ & \multirow[t]{2}{*}{0.657} \\
\hline Poor signet/mucinous & $14(37.8)$ & $3(37.5)$ & $11(37.9)$ & \\
\hline \multicolumn{5}{|l|}{ Tumor length, cm } \\
\hline$>3$ & $31(83.8)$ & $7(87.5)$ & $24(82.8)$ & \multirow[t]{2}{*}{0.613} \\
\hline$\leq 3$ & $6(16.2)$ & $1(3.5)$ & $5(17.2)$ & \\
\hline \multicolumn{5}{|l|}{ Perineural invasion } \\
\hline Present & $15(40.5)$ & $6(75.0)$ & $9(31.0)$ & \multirow[t]{2}{*}{0.034} \\
\hline Absent & $22(59.5)$ & $2(25.0)$ & $20(68.9)$ & \\
\hline \multicolumn{5}{|l|}{ Lymphovascular invasion } \\
\hline Present & $15(40.5)$ & $6(75.0)$ & $9(31.0)$ & \multirow[t]{2}{*}{0.034} \\
\hline Absent & $22(59.5)$ & $2(25.0)$ & $20(68.9)$ & \\
\hline \multicolumn{5}{|l|}{ CRM } \\
\hline Positive $\leq 1 \mathrm{~mm}$ & $4(10.8)$ & $1(12.5)$ & $3(10.3)$ & \multirow[t]{2}{*}{0.640} \\
\hline Negative $>1 \mathrm{~mm}$ & $33(89.2)$ & $7(87.5)$ & $26(89.6)$ & \\
\hline \multicolumn{5}{|l|}{ Mandard classification } \\
\hline TRG2/3 & $24(64.9)$ & $4(50)$ & $20(68.9)$ & \multirow[t]{2}{*}{0.278} \\
\hline TRG4/5 & $13(35.1)$ & $4(50)$ & $9(31.0)$ & \\
\hline
\end{tabular}

Bold values are statistically significant $(p<0.05)$

$C R M$ circumferential resection margin, EMVI extramural venous invasion, AC adenocarcinoma, SCC squamous cell carcinoma, TRG tumor regression grade

9.7\%) and SCCs (47.4\% vs. 19.4\%), as well as in tumors with LVI $(63.2 \%$ vs. $27.4 \%)$. In the nCRT group, the numbers regarding type and tumor side were too small to draw clear conclusions. However, EMVI was significantly higher in tumors with perineural tumor growth and those with LVI-both $75 \%$ vs. $31 \%$, respectively.

More or less the same percentages are found as in CRC, where EMVI is reported in approximately $28 \%$ (range $13.4-31.4 \%$ ) of the resection specimens. ${ }^{6}$ As in CRC, EMVI was common in high T- and N-staged EC. ${ }^{6}$ However, there is wide variability in the assessment of VI. Several reports regarding current practice and agreements among pathologists showed a higher detection rate in university hospitals with experienced gastrointestinal pathologists and the benefits of routine use of additional elastin stain. Moreover, accurate detection and quality in reporting of VI had shown a prognostic significance on cancer survival. $^{14,15}$

Most reports in EC have only described the presence of VI in general, without further distinction of IMVI and EMVI. ${ }^{9,16-18}$ Although EMVI is routinely diagnosed by the presence of key hallmarks, i.e. the 'orphan artery' and 'protruding tongue' signs, accurate detection of EMVI can be problematic. Therefore, separate reporting of venous and small vessel invasion (VI) and notification of EMVI has been recommended in pathological guidelines for CRC. ${ }^{5,19}$ EMVI observation can be hampered when the muscular wall of the veins is obliterated beyond morphologic recognition in specimens after surgery alone or when altered by the use of nCRT due to increased vessel fibrosis and destructed vessel wall architecture. This may result in under-identification. Therefore, elastin stain, known as the 
FIG. 2 a Kaplan-Meier survival curve for disease-free survival stratified by EMVI and lymph node status after surgery alone $(p=0.004)$. b KaplanMeier survival curve for overall survival stratified by EMVI and lymph node status after surgery alone $(p=0.002)$. EMVI extramural venous invasion, Cum cumulative

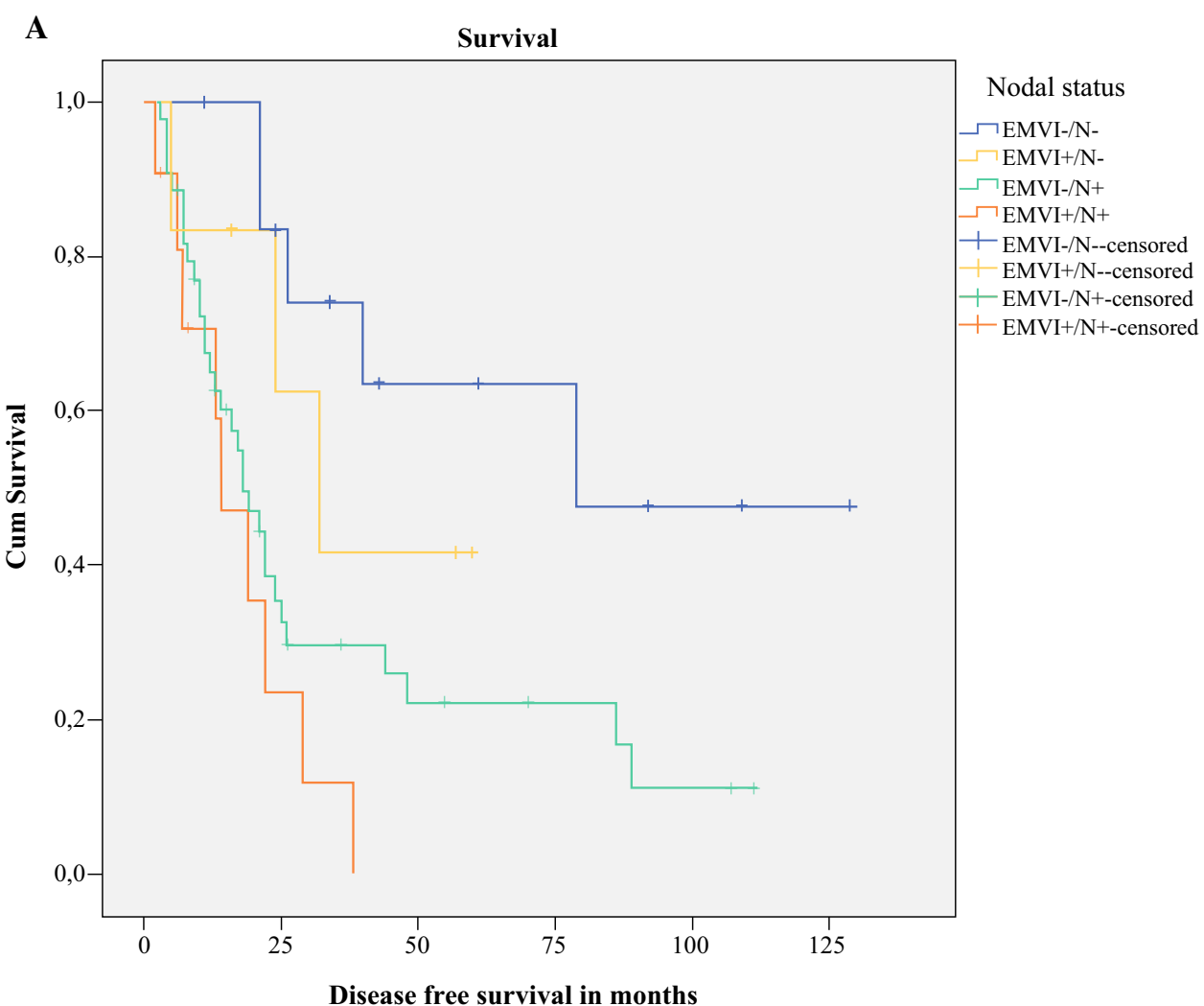

B

Survival

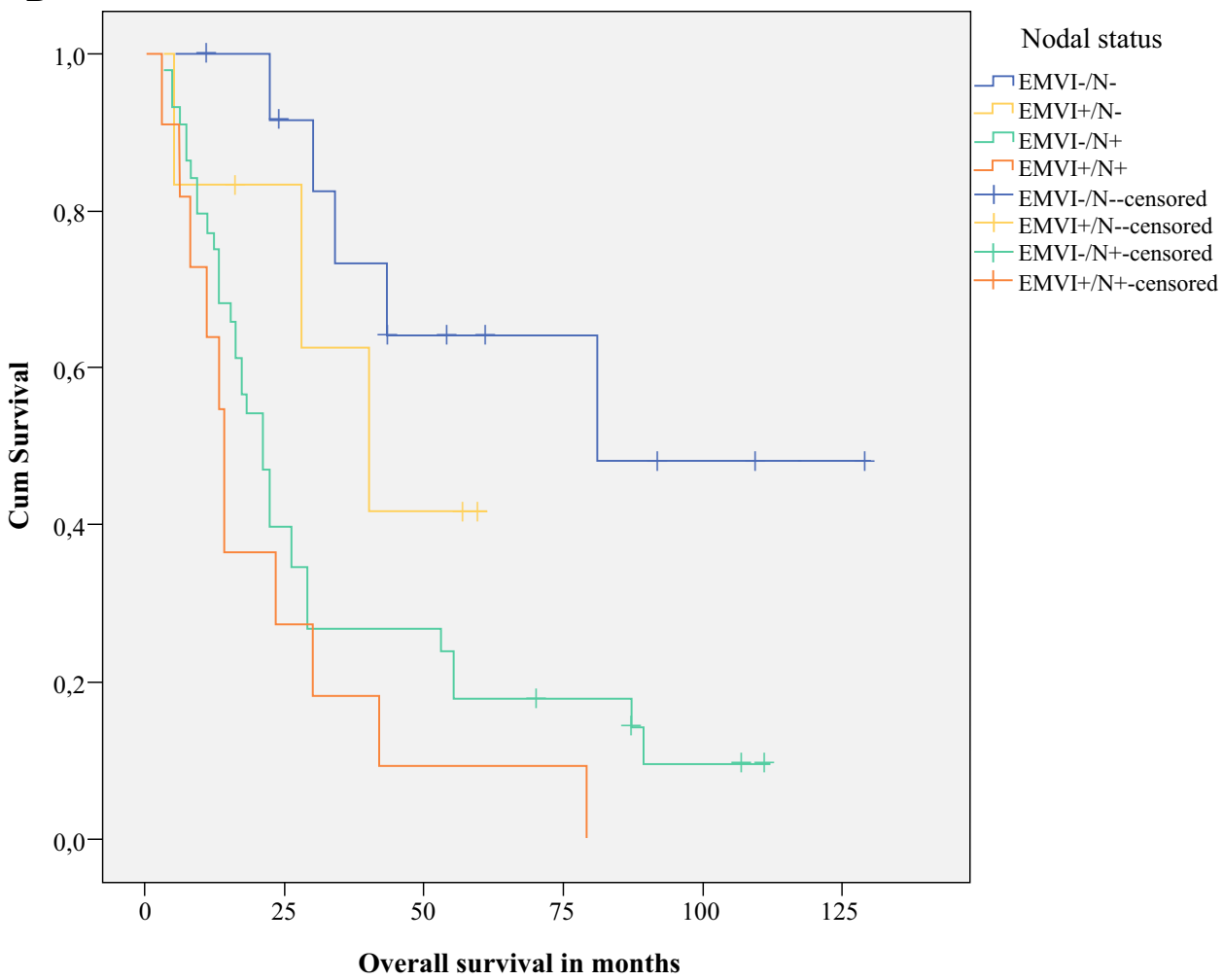

EVG of vessel walls, is generally advocated to solve the majority of these difficult cases. ${ }^{13}$ EVG stain judged as absent or present has shown to double the EMVI detection rates, with increased interobserver agreement between pathologists. $^{19-21}$ 
TABLE 5 Univariable analyses with regard to DFS and OS in 34 patients with pT3 esophageal cancer after neoadjuvant chemoradiotherapy

\begin{tabular}{|c|c|c|c|c|}
\hline \multirow[t]{2}{*}{ Factor } & \multicolumn{2}{|l|}{ DFS } & \multicolumn{2}{|l|}{ OS } \\
\hline & $\mathrm{HR}(95 \% \mathrm{CI})$ & $p$ value & $\mathrm{HR}(95 \% \mathrm{CI})$ & $p$ value \\
\hline Sex (male/female) & $0.7(0.2-3.1)$ & 0.653 & $0.8(0.2-3.4)$ & 0.732 \\
\hline Age & $0.2(0.0-1.5)$ & 0.120 & $0.4(0.1-1.9)$ & 0.267 \\
\hline Tumor length $(\leq 3 />3 \mathrm{~cm})$ & $1.1(0.3-4.0)$ & 0.942 & $1.4(0.4-4.9)$ & 0.568 \\
\hline Localization (mid/distal) & $0.5(0.1-4.1)$ & 0.557 & $0.4(0.0-2.8)$ & 0.327 \\
\hline Histology (SCC/AC) & $0.5(0.1-4.0)$ & 0.544 & $0.6(0.0-4.2)$ & 0.327 \\
\hline pN1-3 & $1.9(0.8-4.6)$ & 0.157 & $2.0(0.8-5.0)$ & 0.129 \\
\hline Differentiation grade (good/moderate vs. poor/signet cell/mucinous) & $1.0(0.4-2.3)$ & 0.942 & $1.0(0.4-2.5)$ & 0.966 \\
\hline Perineural growth (present/absent) & $1.1(0.5-2.6)$ & 0.840 & $1.2(0.5-3.0)$ & 0.656 \\
\hline Lymphovascular invasion (present/absent) & $1.1(0.4-2.5)$ & 0.885 & $0.9(0.4-2.3)$ & 0.879 \\
\hline CRM (positive/negative) & $2.3(0.7-7.3)$ & 0.155 & $3.7(1.1-12.4)$ & $\mathbf{0 . 0 3 4}$ \\
\hline EMVI (present/absent) & $1.1(0.4-3.1)$ & 0.829 & $0.9(0.3-2.9)$ & 0.955 \\
\hline EMVI/N (nodal status) & $1.3(0.9-19)$ & 0.173 & $1.3(0.9-2.0)$ & 0.174 \\
\hline EMVI $-/ \mathrm{N}-$ & Ref. & & Ref. & \\
\hline $\mathrm{EMVI}+/ \mathrm{N}-$ & $0.9(0.2-4.2)$ & 0.997 & $1.0(0.2-4.9)$ & 0.961 \\
\hline EMVI $-/ \mathrm{N}+$ & $1.8(0.7-4.9)$ & 0.236 & $2.1(0.8-5.7)$ & 0.148 \\
\hline $\mathrm{EMVI}+/ \mathrm{N}+$ & $2.1(0.5-8.3)$ & 0.287 & $1.8(0.4-9.1)$ & 0.463 \\
\hline Mandard classification TRG2/3/TRG4/5 & $1.4(0.6-3.4)$ & 0.401 & $1.2(0.5-3.0)$ & 0.667 \\
\hline
\end{tabular}

Bold indicates $p$ value $<0.2$ in univariable analysis were included in the Cox regression multivariable analysis

$D F S$ disease-free survival, $O S$ overall survival, $p$ pathological, $C R M$ circumferential resection margin, EMVI extramural venous invasion, $H R$ hazard ratio, $C I$ confidence interval, $A C$ adenocarcinoma, SCC squamous cell carcinoma, TRG tumor regression grade

TABLE 6 Multivariable analyses (backward conditional Cox regression model) with regard to DFS and OS in 34 patients with pT3 esophageal cancer after neoadjuvant chemoradiotherapy

\begin{tabular}{|c|c|c|c|c|}
\hline \multirow[t]{2}{*}{ Factor } & \multicolumn{2}{|l|}{ DFS } & \multicolumn{2}{|l|}{ OS } \\
\hline & HR $(95 \% \mathrm{CI})$ & $p$ value & HR $(95 \% \mathrm{CI})$ & $p$ value \\
\hline Age & $0.2(0.0-1.5)$ & 0.120 & - & \\
\hline pN1-3 & $1.3(0.9-2.0)$ & 0.174 & $1.6(1.1-2.5)$ & $\mathbf{0 . 0 2 0}$ \\
\hline CRM (positive/negative) & $1.4(0.4-5.3)$ & 0.572 & $2.1(0.6-7.9)$ & 0.272 \\
\hline EMVI/N (nodal status) & $0.7(0.3-1.9)$ & 0.524 & $0.7(0.3-1.6)$ & 0.460 \\
\hline EMVI -/N - & Ref. & & Ref. & \\
\hline $\mathrm{EMVI}+/ \mathrm{N}-$ & $1.1(0.2-5.4)$ & 0.888 & $1.0(0.2-4.9)$ & 0.967 \\
\hline EMVI $-/ \mathrm{N}+$ & $0.5(0.1-3.9)$ & 0.512 & $0.3(0.0-3.5)$ & 0.366 \\
\hline EMVI +/N + & $0.6(0.1-5.4)$ & 0.692 & $0.3(0.0-3.9)$ & 0.382 \\
\hline
\end{tabular}

Bold value is statistically significant $(p<0.05)$

DFS disease-free survival, $O S$ overall survival, $p$ pathological, $C R M$ circumferential resection margin, EMVI extramural venous invasion, $H R$ hazard ratio, $C I$ confidence interval

For the identification of VI in esophageal AC, Castonguay et al. used Movat stains, which is more or less equal to EVG staining. ${ }^{9}$ Both are useful for visualizing connective tissue and elastic fibers. Due to the damage and loss of endothelium, immunohistochemistry, including CD31 and CD34, is often inconclusive. However, in some instances, the only residual clue of VI is a layer of elastin around a round nest of neoplastic cells, which can only be confirmed reliably with elastin stain. ${ }^{21-24}$
As in CRC, we found that EMVI in EC should be considered as a potential high-risk factor in developing metastatic disease. Consequently, as in CRC, we also observed a significantly shorter DFS in the surgery-alone group (HR 2.4, 95\% CI 1.1-4.9) in EMVI-positive EC compared with EMVI-negative tumors (median 25 and 48 months); however, in the nCRT group, the presence of EMVI was not independently associated with survival. The clinical utility in this nCRT group was probably lacking 
due to a potential bias from case mix with low squamous cell cancers, therefore EMVI should be investigated in a larger study group. Another explanation may be the power of our study group, which was too small or was based on the selection of non-responders to nCRT. Failure of EMVI to regress after nCRT may indicate a lack of response, as shown by the association of EMVI changes on magnetic resonance imaging (MRI) with survival outcomes in CRC, and a potential positive effect of nCRT on microscopic distant disease. ${ }^{13,25-27}$ Regression of EMVI following neoadjuvant treatment that results in vessel fibrosis can be used as a predictive imaging biomarker in several gastrointestinal cancers. ${ }^{25,26}$ Moreover, in rectal cancer, the presence of MRI-related EMVI (mrEMVI) within or beyond the mesorectal fat was used to identify high-risk patients who may benefit from neoadjuvant treatment, whereas among patients with stage III gastric cancer, contrast-enhanced multiple-row detector CT might also depict EMVI. ${ }^{25-27}$ Whether the presence and grading of EMVI following nCRT can be accurately assessed on radiologic imaging should be investigated in ongoing or future diffusion-weighted imaging/MRI studies.

To our knowledge, this is the first study comparing outcomes of EMVI among pathological T3 or higher stages in a relatively large group of EC resection specimens in a single, high-volume institute. The usual limitations of retrospective studies and the relatively small sample size of EMVI hinder our ability to draw definite conclusions on the significance of EMVI, especially after nCRT. However, there was no difference in histology type for DFS after surgery alone. The prognostic impact of EMVI after nCRT in SCC is still unclear. EMVI is a histopathologic feature associated with an increased risk of recurrences, and, based on our study, it should be considered as a routine part of pathological reports of resection specimens of EC patients. However, its value should be studied prospectively in larger series and following nCRT in different tumor stages and different histological types.

\section{CONCLUSION}

In this study, EMVI was present in $23.5 \%$ of patients in the surgery-alone group in $21.6 \%$ of patients after nCRT. EMVI was an independently adverse prognostic factor in patients after surgery alone. Therefore, EMVI should be considered as part of the routine histopathology workup, with an accessible use of elastin stains.

OPEN ACCESS This article is distributed under the terms of the Creative Commons Attribution 4.0 International License (http:// creativecommons.org/licenses/by/4.0/), which permits unrestricted use, distribution, and reproduction in any medium, provided you give appropriate credit to the original author(s) and the source, provide a link to the Creative Commons license, and indicate if changes were made.

\section{REFERENCES}

1. Matsuda T, Kurokawa Y, Yoshikawa T et al. Clinicopathological characteristics and prognostic factors of patients with siewert type II esophagogastric junction carcinoma: a retrospective multicenter study. World J Surg. 2016;40(7):1672-9.

2. Lagarde SM, Phillips AW, Navidi M, Disep B, Immanuel A, Griffin SM. The presence of lymphovascular and perineural infiltration after neoadjuvant therapy and oesophagectomy identifies patients at high risk for recurrence. $\mathrm{Br} J$ Cancer. 2015;113(10): 1427-33.

3. Rice TW, Patil DT, Blackstone EH. 8th edition AJCC/UICC staging of cancers of the esophagus and esophagogastric junction: application to clinical practice. Ann Cardiothorac Surg. 2017;6(2):119-130.

4. Huang Q, Luo $\mathrm{K}$, Chen $\mathrm{C}$ et al. Identification and validation of lymphovascular invasion as a prognostic and staging factor in node-negative esophageal squamous cell carcinoma. $J$ Thorac Oncol. 2016;11(4):583-92.

5. Betge J, Pollheimer MJ, Lindtner RA et al. Intramural and extramural vascular invasion in colorectal cancer: prognostic significance and quality of pathology reporting. Cancer. 2012;118(3):628-38.

6. McClelland D, Murray GI. A comprehensive study of extramural venous invasion in colorectal cancer. PLOS One. 2015;10(12):e0144987.

7. Talbot IC, Ritchie S, Leighton MH, Hughes AO, Bussey HJ, Morson BC. The clinical significance of invasion of veins by rectal cancer. Br J Surg. 1980;67(6):439-42.

8. Dirschmid K, Sterlacci W, Oellig F et al. Absence of extramural venous invasion is an excellent predictor of metastasis-free survival in colorectal carcinoma stage II-a study using tangential tissue sectioning. J Clin Pathol. 2012;65(7):619-23.

9. Castonguay MC, Li-Chang HH, Driman DK. Venous invasion in oesophageal adenocarcinoma: enhanced detection using elastic stain and association with adverse histological features and clinical outcomes. Histopathology. 2014;64(5):693-700.

10. Courtney ED, West NJ, Kaur C et al. Extramural vascular invasion is an adverse prognostic indicator of survival in patients with colorectal cancer. Colorectal Dis. 2009;11(2):150-6.

11. Suzuki A, Togashi K, Nokubi M, et al. Evaluation of venous invasion by Elastica van Gieson stain and tumor budding predicts local and distant metastases in patients with $\mathrm{T} 1$ stage colorectal cancer. Am J Surg Pathol. 2009;33(11):1601-7.

12. Shapiro J, van Lanschot JJB, Hulshof MCCM, van Hagen P, van Berge Henegouwen MI et al. Neoadjuvant chemoradiotherapy plus surgery versus surgery alone for oesophageal or junctional cancer (CROSS): long-term results of a randomised controlled trial. Lancet Oncol. 2015;16(9):1090-1098.

13. Tang K, Santos LD. "Orphan arteriole" sign - a helpful clue in detecting extramural venous invasion in colorectal carcinomas. Pathology. 2013;45:S85.

14. Roxburgh CS, McMillan DC, Richards $\mathrm{CH}$ et al. The clinical utility of the combination of $\mathrm{T}$ stage and venous invasion to predict survival in patients undergoing surgery for colorectal cancer. Ann Surg. 2014;259(6):1156-65.

15. Messenger DE, Driman DK, McLeod RS, Riddell RH, Kirsch R. Current practice patterns among pathologists in the assessment of venous invasion in colorectal cancer. $J$ Clin Pathol. 2011;64(11):983-9. 
16. Edwards JM, Hillier VF, Lawson RA, Moussalli H, Hasleton PS. Squamous carcinoma of the oesophagus: histological criteria and their prognostic significance. Br J Cancer. 1989;59(3):429-33.

17. Robey-Cafferty SS, el-Naggar AK, Sahin AA, Bruner JM, Ro JY, Cleary KR. Prognostic factors in esophageal squamous carcinoma. A study of histologic features, blood group expression, and DNA ploidy. Am J Clin Pathol. 1991;95(6):844-9.

18. Sugimachi K, Matsuura H, Kai H, Kanematsu T, Inokuchi K, Jingu K. Prognostic factors of esophageal carcinoma: univariate and multivariate analyses. J Surg Oncol. 1986;31(2):108-12.

19. Dawson H, Kirsch R, Driman DK, Messenger DE, Assarzadegan $\mathrm{N}$, Riddell RH. Optimizing the detection of venous invasion in colorectal cancer: the Ontario, Canada, experience and beyond. Front Oncol. 2015;4:354.

20. Howlett CJ, Tweedie EJ, Driman DK. Use of an elastic stain to show venous invasion in colorectal carcinoma: a simple technique for detection of an important prognostic factor. $J$ Clin Pathol. 2009;62(11):1021-5.

21. Vass DG, Ainsworth R, Anderson JH et al. The value of an elastic tissue stain in detecting venous invasion in colorectal cancer. $J$ Clin Pathol. 2004;57;769-772.

22. Kirsch R, Messenger DE, Riddell RH et al. Venous invasion in colorectal cancer: impact of an elastin stain on detection and interobserver agreement among gastrointestinal and nongastrointestinal pathologists. Am J Surg Pathol. 2013;37;200-210.

23. Movat HZ. Demonstration of all connective tissue elements in a single section; pentachrome stains. AMA Arch Pathol. 1955;60(3):289-95.

24. Sigdel S, Gemind JT, Tomashefski JF Jr. The Movat pentachrome stain as a means of identifying microcrystalline cellulose among other particulates found in lung tissue. Arch Pathol Lab Med. 2011;135(2):249-54.

25. Chand M, Swift RI, Tekkis PP, Chau I, Brown G. Extramural venous invasion is a potential imaging predictive biomarker of neoadjuvant treatment in rectal cancer. $\mathrm{Br} J$ Cancer. 2014;110(1):19-25.

26. Chand M, Bhangu A, Wotherspoon A et al. EMVI-positive stage II rectal cancer has similar clinical outcomes as stage III disease following pre-operative chemoradiotherapy. Ann Oncol. 2014;25(4):858-63.

27. Cheng J, Wu J, Ye Y, Zhang C, Zhang Y, Wang Y. The prognostic significance of extramural venous invasion detected by multiple-row detector computed tomography in stage III gastric cancer. Abdom Radiol (NY). 2016;41(7):1219-26. 